\title{
Mudas de Mimosa caesalpiniaefolia Benth (Leguminosae: Mimosoideae) cultivadas em substratos orgânicos
}

\section{Seedlings of Mimosa caesalpiniaefolia Benth (Leguminosae: Mimosoideae) grown on organic substrates}

\author{
José Israel Pinheiro ${ }^{1}$, Lucas de Sousa Oliveira ${ }^{2}$, Alfredo Mendonça de Sousa ${ }^{3}$, Kaio Gráculo Vieira Garcia ${ }^{4}$, Luan Alves Lima ${ }^{5}$
}

Resumo: O sucesso no cultivo de espécies florestais, como o sabiá (Mimosa caesalpiniaefolia Benth), depende, essencialmente, da utilização de mudas de boa qualidade. Nessa etapa da produção, diversos materiais possuem potencial de uso, principalmente, resíduos orgânicos, notando-se, contudo, pouca informação a respeito do potencial de utilização desses resíduos na produção de mudas. Diante do exposto, objetivou-se avaliar o desenvolvimento inicial de mudas de sabiá em função da utilização de substratos orgânicos. O experimento foi conduzido em casa de vegetação pertencente ao Departamento de Ciências do Solo da Universidade Federal do Ceará, Fortaleza, Ceará. O delineamento experimental foi inteiramente casualizado com quatro tratamentos, esterco bovino + areia (E.B), esterco caprino + areia (E.Ca), esterco de coelho + areia (E.Co), todos na proporção de 1:1, e controle a base de areia (A) com seis repetições. Aos 84 dias após a semeadura as plantas foram coletadas para a determinação das seguintes variáveis: número de folhas (NF), altura de plantas (ALT), diâmetro do caule (DC), comprimento da raiz (CR), massa seca da parte aérea (MSPA) e massa seca de raiz (MSR). Com exceção da variável $\mathrm{CR}$, todas as variáveis foram influenciadas significativamente pelo uso dos substratos. O NF apresentou maiores médias quando as plantas foram cultivadas em esterco de caprino ou bovino. Em relação à ALT e DC, constatou-se comportamento semelhante ao NF, no entanto, para o DC, o esterco de coelho foi estatisticamente semelhante ao bovino e caprino. Assim, mudas de sabiá podem ser produzidas em substrato base de estercos bovino e caprino em mistura com areia na proporção de 1:1. O melhor desenvolvimento inicial de mudas de sabiá se dá com a utilização de substrato à base de esterco bovino.

Palavras-chave: Adubação orgânica; Espécies florestais; Sustentabilidade.

\begin{abstract}
Success in growing forest species, such as sage (Mimosa caesalpiniaefolia Benth), depends essentially on the use of good quality seedlings. At this stage of production, several materials have potential for use, mainly, organic waste. However, there is little information about the potential for the use of these residues in the production of seedlings. In view of the above, the objective was to evaluate the initial development of sapiens' seedlings due to the use of organic substrates. The experiment was conducted in a greenhouse belonging to the Soil Science Department (SSD) of the Federal University of Ceará , Fortaleza, Ceará. The experimental design was completely randomized with four treatments, as follows: bovine manure + sand (BM), goat manure + sand $(\mathrm{GM})$, rabbit manure + sand $(\mathrm{RM})$, all in a ratio of 1: 1 , and control the sand base (S) with six replicates. At 84 days after sowing the plants were collected for the determination of the following variables: leaf number (LN), plant height (PH), stem diameter (SD), root length (RL), shoot dry mass (SDM) and root dry mass (RDM). With the exception of the RL variable, all variables were significantly influenced by the use of the substrates. LN showed higher averages when grown in goat or bovine manure. In relation to PH and SD, LN-like behavior was observed, however, for SD, rabbit manure was statistically similar to bovine and goat. Thus, sage seedlings can be produced on a substrate basis of bovine and goat manure in a mixture with sand in a ratio of 1: 1 . The best initial development of sage saplings occurs with the use of substrate based on bovine manure.
\end{abstract}

Key words: Organic fertilization; Forest species; Sustainability.

\footnotetext{
*Autor para correspondência

Recebido para publicação em 05/02/2018; aprovado em 20/03/2018

${ }^{1}$ Doutorando em Ciência do Solo, Universidade Federal do Ceará, E-mail: jisraelpinheiro@ gmail.com

${ }^{2}$ Doutorando em Ciência do Solo, Universidade Federal do Ceará, E-mail: eng.luccas@ hotmail.com

${ }^{3}$ Doutorando em Engenharia Agrícola/Universidade Federal do Ceará, E-mail: alfredomendonca121@gmail.com

${ }^{4}$ Doutorando em Ciência do Solo, Universidade Federal do Ceará, E-mail: kaiovieira88@ @otmail.com

${ }^{5}$ Doutorando em Ciência do Solo, Universidade Federal do Ceará, E-mail: luanefa2@ yahoo.com.br*
} 


\section{INTRODUÇÃO}

O sabiá (Mimosa caesalpiniaefolia Benth) é uma espécie leguminosa da família Mimosaceae que, dependendo da região, pode ser conhecida também por sansão-do-campo. Sua ocorrência natural é comum na região Nordeste, principalmente, nos estados do Rio Grande do Norte, Piauí e Ceará, predominando no bioma Caatinga que integra esses estados. É considerada uma espécie precoce, heliófila, pioneira e seletiva xerófila, adapta-se em todos os tipos de solo, com exceção feita a condições de várzeas, devido ao alagamento, podendo ser propagada via sementes, estacas ou rebrotas de troncos e raízes (GARCIA et al., 2016).

Essa leguminosa apresenta sistema radicular profundo, embora concentre o maior volume de raízes nos primeiros 20 $\mathrm{cm}$ de profundidade, favorecendo, dessa forma, a absorção de água e auxiliando o desenvolvimento da espécie em condições ambientais limitantes (PARDON et al., 2017).

A planta possui alto poder calorífico e resistência mecânica em sua madeira, com isso, é grande o valor econômico agregado, tendo sido usada como alternativa energética e, principalmente, para a produção de estacas no Nordeste brasileiro.

Além disso, o Sabiá apresenta potencial como espécie forrageira; sua biomassa contém em média $17 \%$ de proteína bruta, além das vagens que são excelente fonte de alimento para ruminantes no período de chuvas escassas (CHOTCHUTIMA et al., 2016). Nos últimos anos tem sido utilizada em diversos estudos envolvendo a recuperação de áreas degradadas (GONZAGA et al., 2016) e contaminadas por metais pesados, principalmente em função de suas características morfológicas (SCHNEIDER et al., 2017).

O sucesso no estabelecimento de espécies florestais no campo está associado à produção de mudas de boa qualidade. Diversos são os materiais passíveis de uso no processo de confecção de substratos para produção de mudas, uma tendência que merece destaque é a utilização de resíduos orgânicos misturados ao solo, que, além dos nutrientes, destacam-se por ser fonte de matéria orgânica, o que contribui para melhoria das propriedades físicas, químicas e biológicas do substrato e proporciona melhores condições ao desenvolvimento das raízes das plantas e das mudas (GASPARIN et al., 2014). Mesmo com toda importância que a espécie tem para a região Nordeste, o conhecimento sobre a produção de mudas de sabiá ainda é incipiente, como ocorre também para a maioria das espécies florestais, fazendo-se necessário, portanto, aplicação de técnicas que possibilitem o adequado desenvolvimento das mudas. Diante do exposto, objetivou-se avaliar o desenvolvimento inicial de mudas de sabiá (Mimosa caesalpiniaefolia Benth) em função da utilização de substratos orgânicos.

\section{MATERIAL E MÉTODOS}

O experimento foi conduzido em casa de vegetação pertencente ao Departamento de Ciências do Solo (DCS) da Universidade Federal do Ceará (UFC) - Campus do Pici, Fortaleza, Ceará.

Os materiais orgânicos foram obtidos da área de compostagem na horta didática da UFC, pertencente ao departamento de Fitotecnia. Ao final da compostagem o material foi selecionado em peneira com abertura de malha de $2 \mathrm{~mm}$, homogeneizado e colocado nos vasos para o plantio das mudas, uma outra parte do material foi levado a secagem em estufa de circulação forçada de ar à temperatura de $65^{\circ} \mathrm{C}$ e, depois de seco, acondicionado em sacos plásticos devidamente identificados.

De cada resíduo foi retirado uma alíquota para envio ao Laboratório de análises físicas e químicas de solo e água, pertencente a Fundação Cearense de Meteorologia FUNCEME, que funciona em parceria com a Universidade Federal do Ceará - UFC, para análise química dos substratos orgânicos (Tabela 1).

Tabela 1. Caracterização química dos substratos orgânicos utilizadas no experimento

\begin{tabular}{ccccc}
\hline \multirow{2}{*}{ Atributos químicos } & \multicolumn{4}{c}{ Substratos orgânicos } \\
\cline { 2 - 5 } & E.B & E.Ca & E.Co & $\mathrm{A}$ \\
\hline $\mathrm{pH}\left(\mathrm{H}_{2} \mathrm{O} \mathrm{1:2,5)}\right.$ & 6,3 & 6,1 & 6,6 & 7,5 \\
$\mathrm{C} . \mathrm{E} .\left(\mathrm{dS} \mathrm{m}^{-1}\right)$ & 7,1 & 5,4 & 2,2 & 0,0 \\
$\mathrm{Ca}\left(\mathrm{cmol}_{\mathrm{c}} \mathrm{kg}^{-1}\right)$ & 7,2 & 13,0 & 11,0 & 0,5 \\
$\mathrm{Mg}\left(\mathrm{cmol}_{\mathrm{c}} \mathrm{kg}^{-1}\right)$ & 1,3 & 7,4 & 8,7 & 0,4 \\
$\mathrm{Na}\left(\mathrm{cmol}_{\mathrm{c}} \mathrm{kg}^{-1}\right)$ & 0,7 & 0,3 & 0,4 & 0,0 \\
$\mathrm{~K}\left(\mathrm{cmol}_{\mathrm{c}} \mathrm{kg}^{-1}\right)$ & 3,2 & 1,9 & 1,7 & 0,0 \\
$\mathrm{H}+\mathrm{Al}\left(\mathrm{cmol}_{\mathrm{c}} \mathrm{kg}^{-1}\right)$ & 3,3 & 4,1 & 4,7 & 0,3 \\
$\mathrm{Al}\left(\mathrm{cmol}_{\mathrm{c} \mathrm{kg}}{ }^{-1}\right)$ & 0,4 & 0,3 & 0,4 & 0,0 \\
$\mathrm{~S}\left(\mathrm{cmol}_{\mathrm{c}} \mathrm{kg}^{-1}\right)$ & 12,6 & 22,7 & 21,9 & 1,0 \\
$\mathrm{P}\left(\mathrm{cmol}_{\mathrm{c}} \mathrm{kg}^{-1}\right)$ & 2082 & 1988 & 4705 & 28,0 \\
$\mathrm{CO}\left(\mathrm{g} \mathrm{kg}^{-1}\right)$ & 54,6 & 52,0 & 49,8 & 0,3 \\
$\mathrm{~N}\left({\left.\mathrm{~g} / \mathrm{kg} \mathrm{g}^{-1}\right)}_{\mathrm{T}}^{5,5}\right.$ & 5,4 & 5,2 & 0,0 \\
$\mathrm{~V} \%$ & 15,9 & 26,0 & 26,7 & 1,3 \\
$\mathrm{~m} \%$ & 79,0 & 86,0 & 82 & 77,0 \\
$\mathrm{PST}$ & 3,0 & 2,0 & 2,0 & - \\
$\mathrm{C} / \mathrm{N}$ & 5,0 & 2,0 & 2,0 & 5,0 \\
\hline
\end{tabular}

C.E: Condutividade elétrica; $\mathrm{H}+\mathrm{Al}$ : Acidez potencial; $\mathrm{CO}$ : Carbono orgânico; T: Capacidade de troca de cátions; V: Saturação por bases; m: Saturação por alumínio; PST: Saturação por sódio; C/N: Relação carbono nitrogênio; E.B: Esterco bovino; E.Ca: Esterco caprino; E.Co: Esterco de coelho; A: Controle a base de areia.

O delineamento experimental utilizado foi inteiramente casualisado com quatro tratamentos e seis repetições, totalizando 24 unidades experimentais. Os tratamentos foram compostos pelos seguintes substratos: esterco bovino + areia (E.B), esterco caprino + areia (E.Ca), esterco de coelho + areia (E.Co), todos na proporção de 1:1, e Controle a base de areia (A). Cada unidade experimental foi representada por um vaso plástico com capacidade de $2 \mathrm{~L}$, contento uma planta por cada vaso.

As sementes de sabiá foram adquiridas da empresa Biosementes e a semeadura foi realizada no dia 02 de agosto de 2016, colocando-se duas sementes por vaso, sendo feito o desbaste aos 25 dias após a semeadura (DAS), deixando-se uma planta por vaso atribuindo o critério daquela planta mais vigorosa. O suprimento de água foi feito por meio de irrigação manual, diariamente, sempre no período da tarde. Durante esse período não foi feita adubação de cobertura.

Aos 84 DAS às plantas foram coletadas e determinadas as seguintes variáveis: número de folhas (NF), altura de plantas (ALT), diâmetro do caule (DC), comprimento da raiz (CR), massa seca da parte aérea (MSPA) e massa seca de raiz (MSR).

Para o número de folhas foi considerado somente aquelas folhas totalmente definidas. A altura de planta foi determinada utilizando régua graduada, medindo-se desde o 
colo da planta até o ápice da maior folha. O diâmetro do caule foi obtido com auxílio de paquímetro digital $(0,01 \mathrm{~mm})$, sendo as medidas tomadas a $1 \mathrm{~cm}$ da superfície do solo dentro do vaso.

Foram feitas medidas biométricas de comprimento de raiz nas plantas utilizando-se de régua graduada, em seguida as plantas foram lavadas, separadas (raiz e parte aérea) e colocadas cada uma em saco de papel para acondicionamento em estufa de circulação forçada de ar à temperatura de $65^{\circ} \mathrm{C}$ até atingir peso constante para, posteriormente, determinar o peso seco utilizando-se balança digital analítica $(0,01 \mathrm{~g})$.

Os dados foram submetidos a analise de variância (ANOVA) e as variáveis que apresentaram resposta significativa foram comparados entre si pelo teste de Tukey ao nível de $5 \%$ de probabilidade, utilizando o programa estatístico SISVAR 5.6 (FERREIRA, 2014).

\section{RESULTADOS E DISCUSSÃO}

A partir da realização da ANOVA verificou-se efeito significativo dos tratamentos analisados, ao nível de $1 \%$ de significância, sobre as seguintes variáveis: número de folhas (NF), altura de planta (ALT), diâmetro do caule (DC) e massa seca da parte aérea (MSPA). Para a massa seca da raiz (MSR) houve efeito significativo ao nível de $5 \%$ de probabilidade. Por outro lado, para o comprimento da raiz (CR) não se verificou diferença estatística para os substratos estudados.

Analisando-se o efeito dos substratos sobre o número de folhas (NF) as maiores médias observadas foram nos tratamentos constituídos por esterco de caprino e bovino (Figura 1A). Em relação à altura de planta (ALT) e diâmetro do caule (DC), constatou-se comportamento semelhante a variável anterior, no entanto, para o diâmetro do caule o esterco de coelho foi estatisticamente semelhante ao bovino e caprino (Figuras 1B e 1C).

Pelos resultados expostos na figura $1 \mathrm{~A}$, observou-se que o número médio de folhas nos tratamentos com esterco caprino (E.Ca) e bovino (E.B) foram estatisticamente iguais. Não foi observada diferença significativa entre os tratamentos E.B, A e E.Co (Figura 1A). O número de folhas é um parâmetro usado na seleção das melhores mudas além de ser determinante na percepção da capacidade fotossintética das plantas e da área de transpiração (GASPARIN et al., 2014).

Para a variável altura das plantas, este resultado pode estar relacionado aos níveis de nitrogênio e fósforo encontrados nas misturas. Segundo Faria et al. (2016), nitrogênio e fosforo são nutrientes que mais comumente limitam o crescimento das mudas na fase inicial de produção, uma vez que, teores elevados de $\mathrm{N}$ e $\mathrm{P}$ são altamente requeridos nos estádios iniciais de crescimento da parte aérea.

Nota-se que os teores de $\mathrm{N}, \mathrm{P}$, e Mg (Tabela 1) nos tratamentos E.B, E.Ca e E.Co, podem contribuír para o crescimento das mudas de sabiá, outros estudos com espécies arbóreas nativas verificaram aumentos na altura de plantas e na MSPA com a presença elevada desses nutrientes (MACHADO et al., 2014; FARIA et al., 2016; LEAL et al., 2016). Tais repostas podem está associadas às funções que esses elementos desempenham para o desenvolvimento vegetal, atuando nos processos enzimáticos, transferência de energia, crescimento e alongamento celular (TAIZ et al., 2017).
Figura 1. Número de folhas (A), altura de plantas (B) e diâmetro do caule (C) de mudas de sabiá em diferentes substratos orgânicos [Médias seguidas pela mesma letra minúscula, não diferem entre si pelo teste de Tukey a 5\% de probabilidade]. (esterco bovino - E.B; areia - A; esterco caprino - E.Ca e; esterco de coelho - E. Co)
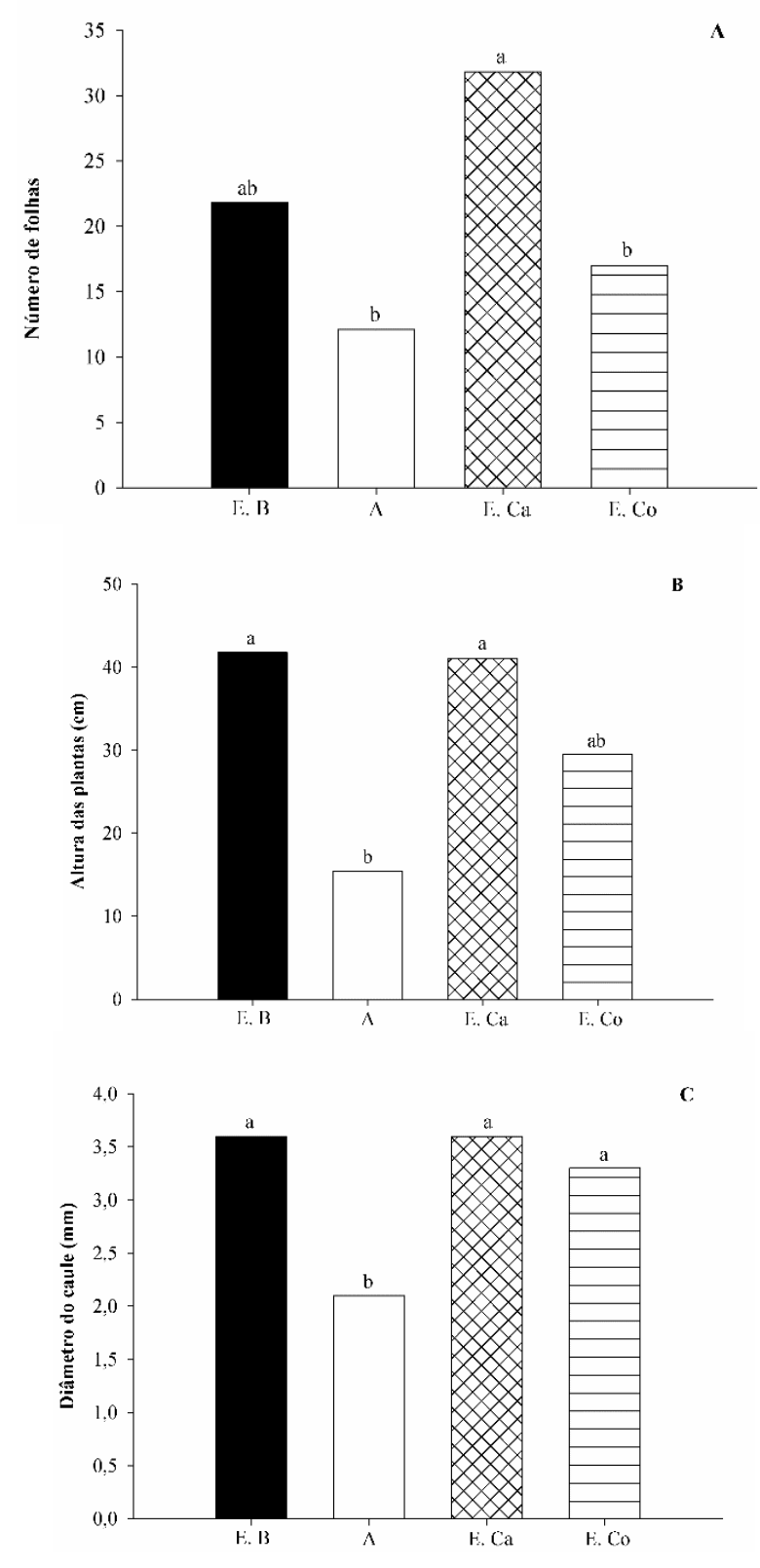

De maneira geral, os parâmetros morfológicos comumente utilizados na determinação intuitiva do padrão de qualidade das mudas, sendo necessária uma definição mais precisa que considere os aspectos relacionados sobrevivência e ao crescimento, determinadas pelas adversidades encontradas no campo após o plantio (GOMES et al., 2002).

Estes resultados são relevantes do ponto de vista das condições do semiárido, uma vez que, o uso de materiais orgânicos é de fácil acesso na maioria das propriedades rurais, desempenhando papel fundamental na recuperação de áreas degradadas por meio da produção de mudas para preservação de espécies da caatinga. Além disso, torna-se uma alternativa promissora à adubação mineral, o que representa economia aos agricultores, aproveitamento desses resíduos, bem como ganhos ambientais (KRATKA; CORREIA, 2015). 
Observou-se pela Figura 1C que não há diferença significativa entre os tratamentos com esterco bovino (E.B), caprino (E.Ca) e de coelho (E.Co), para os valores médios referentes ao diâmetro do caule das plantas de sabiá. Por outro lado, no substrato que continha em sua composição apenas areia foi observado valores inferiores de diâmetro do caule em relação aos demais.

Kratka e Correia (2015) avaliando crescimento inicial de aroeira do sertão (Myracrodruon urundeuva AllemãoAnacardiaceae), observaram que as plantas cultivadas no tratamento contendo esterco bovino apresentaram maiores diâmetros do coleto e que, de maneira geral, as demais variáveis avaliadas apresentaram os melhores resultados sob efeito desse tratamento, corroborando com os resultados observados nesta pesquisa.

Em relação à massa seca da parte aérea (MSPA), observaram-se maiores valores quando as plantas foram cultivadas com esterco caprino, não diferindo estatisticamente do esterco bovino (Figura 2). Os menores valores observados foram os do tratamento controle, que não diferiram estatisticamente do tratamento com esterco de coelho. Observaram-se maiores valores médios de matéria seca da parte aérea em mudas de sabiá cultivadas nos substratos E.Ca e E.B (Figura 2).

Figura 2. Massa seca da parte aérea (MSPA) de mudas de sabiá em diferentes substratos orgânicos [Médias seguidas pela mesma letra minúscula, não diferem entre si pelo teste de Tukey a 5\% de probabilidade]. (esterco bovino - E. B; areia A; esterco caprino - E. Ca e; esterco de coelho - E. Co)

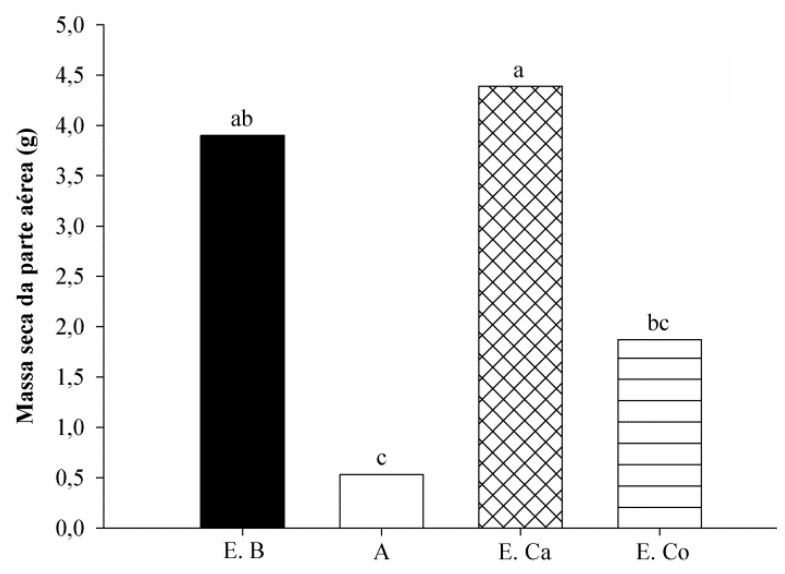

Segundo Leal et al. (2016), esse fato está relacionado à retenção de água encontrada nesses substratos, característica que favorece o desenvolvimento da plântula, conforme observado nesse estudo para essa variável.

Para a massa seca da raiz (MSR) o esterco bovino mostrou melhor resultado, apesar de não diferir, estatisticamente, do esterco caprino (Figura 3).

A MSPA e MSR são dois parâmetros fundamentais e utilizados como critério para a determinação da qualidade de mudas (FRARIAS et al., 2016), sendo essas variáveis relacionadas às atividades fisiológicas das mudas e na interação dos fatores solo-água-planta e ambiente (CARNEIRO, 1995).
Figura 3. Massa seca da raiz (MSR) de mudas de sabiá em diferentes substratos orgânicos [Médias seguidas pela mesma letra minúscula, não diferem entre si pelo teste de Tukey a 5\% de probabilidade]. (esterco bovino - E. B; areia - A; esterco caprino - E. Ca e; esterco de coelho - E. Co)

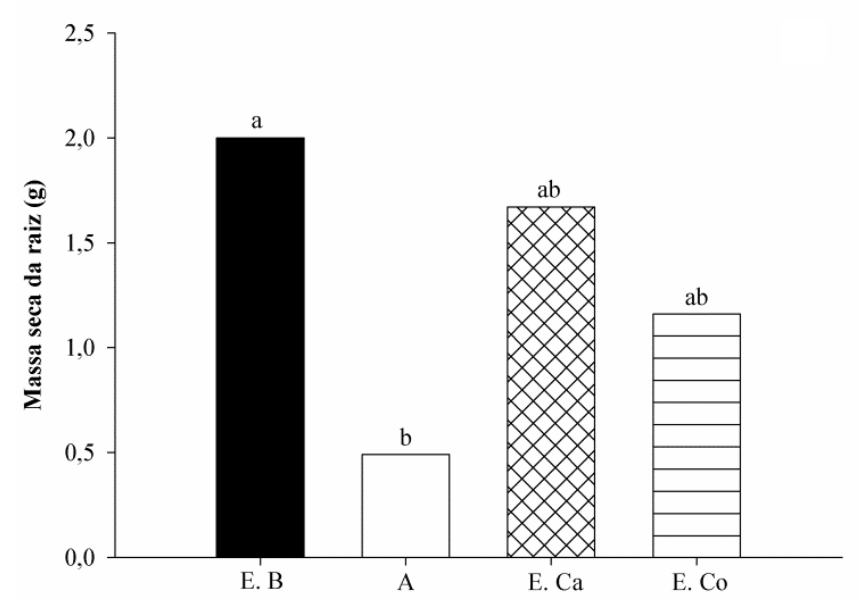

O comportamento das variáveis, massa seca da parte aérea e massa seca da raiz, estão de acordo com resultados obtidos por Minami (1995), afirmando que substratos com maiores concentrações de cálcio e fósforo propiciam condições mais adequadas para o desenvolvimento inicial de plantas, comprovações estas constatadas em todas as variáveis analisadas no presente trabalho, em que, os tratamentos a base de estercos animais, pelos elevados teores desses elementos, foram os que apresentaram maiores valores de massa seca da parte aérea e raiz. Andrade et al. (2015), relatam que a fitomassa de raízes é variável fundamental para produção de mudas, uma vez que condiciona a capacidade da planta em particionar fotoassimilados às raízes, contribuindo para o seu crescimento.

\section{CONCLUSÕES}

Mudas de sabiá podem ser produzidas em substrato base de estercos bovino e caprino em mistura com areia na proporção de 1:1.

O melhor desenvolvimento inicial de mudas de sabiá se dá com a utilização de substrato à base de esterco bovino.

\section{REFERÊNCIAS}

ANDRADE, F. R; PETTER, F. A; JUNIOR, B. H. M; GONÇALVES, L. G. V; SCHOSSLER, T. R; NÓBREGA, J. C. A. Formulação de substratos alternativos na formação inicial de mudas de ingazeiro. Scientia Agraria ParanaensisSAP, v. 14, p. 234-239, 2015.

CARNEIRO, J. G. A. Produção e controle de qualidade de mudas florestais. Curitiba: Universidade Federal do Paraná, 1995. P. $41-65$.

CHOTCHUTIMA, S.; TUDSRI, S.; KANGVANSAICHOL, K.; SRIPICHITT, P. Effects of sulfur and phosphorus application on the growth, biomass yield and fuel properties of leucaena (Leucaena leucocephala (Lam.) de Wit.) as bioenergy crop on sandy infertile soil. Agriculture and Natural Resources, v. 50, p. 54-59, 2016. 
FARIA, J. C. T.; CALDEIRA, M. V. W.; DELARMELINA, W. M. Substratos alternativos na produção de mudas de Mimosa setosa Benth. Ciência Florestal, v. 26, p. 1075-1086, 2016.

FERREIRA, D. F. Sisvar: a Guide for its Bootstrap procedures in multiple comparisons. Ciência agrotecnologia v.38, n.2, p. 109-112, 2014.

FILGUEIRA, F. A. R. Manual de Oleiricultura: Agrotecnologia moderna na produção e comercialização de hortaliças. Viçosa, 2000, 402p.

GARCIA, K. G. V.; GOMES, V. F. F.; ALMEIDA, A. M. M.; FILHO, P. F. M. Micorrizas arbusculares no crescimento de mudas de sabiá em um substrato proveniente da mineração de manganês. Revista Verde de Agroecologia e Desenvolvimento Sustentável, v. 11, p. 15-20, 2016.

GASPARIN, E.; AVILA, A. L.; ARAUJO, M. M.; FILHO, A. C.; DORNELES, D. U.; FOLTZ, D. R. B. Influencia do substrato e do volume de recipiente na qualidade das mudas de Cabralea canjerana (Vell.) Mart. em viveiro e no campo. Ciência Florestal, v. 24, p. 553-563, 2014.

GOMES, J. M; COUTO, L; LEITE, H. G; XAVIER, A.; GARCIA, S. L. R Qualidade dos parâmetros morfológicos para a avaliação de plântulas de Eucalyptus grandis. Revista Árvore, v.26, p.655-664, 2002.

GONÇALVES, F. G.; ALEXANDRE, R. S.; SILVA, A. G.; LEMES, E. Q.; ROCHA, A. P.; RIBEIRO, M. P. A. Emergência e qualidade de mudas de Enterolobium contortisiliquum (Vell.) Morong (Fabaceae) em diferentes substratos. Revista Árvore, v. 37, p. 1125-1133, 2013.

GONZAGA, L. M.; SILVA, S. S.; CAMPOS, S. A.; FERREIRA, R. P.; CAMPOS, A. N. R.; CUNHA, A. C. M. C. M. Recipientes e substratos para a produção de mudas de jatobá (Hymenaea courbaril L.). Revista Brasileira de Agropecuária Sustentável, v. 6, p. 64-73, 2016.

KRATKA, P. C.; CORREIA, C. R. M. A. Crescimento inicial de aroeira do sertão (Myracrodruon urundeuva Allemão) em diferentes substratos. Revista Árvore, v. 39, p. 551-559, 2015.

LEAL, C. C. P.; TORRES, S. B.; BRITO, A. A. F.; FREITAS, R. M. O.; NOGUEIRA, N. W. Emergência e desenvolvimento inicial de plântulas de Cassia grandis L. f. em função de diferentes substratos. Ciência Florestal, v. 26, p. 727-734, 2016.

MACHADO, K. S.; MALTONI, K. L.; SANTOS, C. M.; CASSIOLATO, A. M. R. Resíduos organicos e fósforo como condicionadores de solo degradado e efeitos sobre o crescimento incial de Dipteryx alata Vog. Ciência Florestal, v. 24, p. 541-552, 2014.

MINAMI, K. Produção de mudas de alta qualidade em horticultura. São Paulo; T. A. Queiroz, 1995.
PARDON, P.; REUBENS, B.; REHEUL, D.; MERTENS, J.; DE FRENNE, P.; COUSSEMENT, T.; JANSSENS, P.; VERHEYEN, K. Trees increase soil organic carbon and nutrient availability in temperate agroforestry systems. Agriculture, Ecosystems and Environment, v. 247, p. 98-111, 2017.

SCHNEIDER, V. A. GRAVES-LINDSAY, T.; HOWE, K.; BOUK, N., CHEN, H. C.; KITTS, P. A.; MURPHY, T. D.; PRUITT, K. D.; THIBAUD-NISSEN, F.; ALBRACHT, D.; FULTON, R. S.; KREMITZKI, M.; MAGRINI, V.; MARKOVIC, C.; McGRATH, S.; STEINBERG, K. M.; AUGER, K.; CHOW, W.; COLLINS, J.; HARDEN, G.; HUBBARD, T.; PELAN, S.; SIMPSON, J. T.; THREADGOLD, G.; TORRANCE, J.; WOOD, J. M.; CLARKE, L.; KOREN, S.; BOITANO, M.; PELUSO, P.; LI, H.; CHIN, C. S.; PHILLIPPY, A. M.; DURBIN, R.; WILSON, R. K.; FLICEK, P.; EICHLER, E. E.; CHURCH, D. M. Evaluation of GRCh38 and de novo haploid genome assemblies demonstrates the enduring quality of the reference assembly. Genome Research, v. 27, p. 849-864, 2017.

TAIZ, L.; ZEIGER, E.; MØLLER, I. M.; MURPHY, A. Fisiologia e Desenvolvimento Vegetal. 6.ed. Porto Alegre: Artemed, 2017. 888p.

TAVARES, S. R. L.; FRANCO, A. A.; SILVA, E. M. R. Resposta de sabiá Mimosa caesalpiniaefolia Benth. a incoculação com rizóbio e micorriza em diferentes níveis de fósforo em solo de restinga degradado. Holos, v. 4, p. 1-8, 2016. 